\title{
Estrutura populacional de Xylopia aromatica (Lam.) Mart. e de Roupala montana Aubl. em fragmentos de cerrado no Estado de São Paulo
}

\author{
ANELIZA DE ALMEIDA MIRANDA-MELO ${ }^{1}$, FERNANDO ROBERTO MARTINS ${ }^{2} \mathrm{e}$ \\ FLAVIO ANTONIO MAËS DOS SANTOS 2,3
}

(recebido: 10 de agosto de 2006; aceito: 27 de setembro de 2007)

\begin{abstract}
Population structure of Xylopia aromatica (Lam.) Mart. and of Roupala montana Aubl. in "cerrado" fragments in São Paulo State). In order to understand whether and how population structure of different species varies among different fragments and within the same fragment, we studied the ontogenetic stage and size distributions of two common "cerrado" tree species, in four "cerrado" fragments with different physiognomies and in three sites with similar physiognomies within the same fragment in the Municipality of Itirapina ( $22^{\circ} 15^{\prime}$ S, $47^{\circ} 48^{\prime}$ W), São Paulo state, SE Brazil. We recorded the height and the stem basal diameter (DSH) of all individuals with DSH $\geq 3 \mathrm{~cm}$ in the six samples of 200 contiguous $5 \times 5 \mathrm{~m}$ plots. Individuals with DSH $<3 \mathrm{~cm}$ were sampled in $1 \mathrm{~m}^{2}$ sub-plots at one corner of the plots. Xylopia aromatica had greater proportions of initial ontogenetic stages in open physiognomies, whereas Roupala montana had high proportion of initial stages and greater young:adult ratio in the densest physiognomy. The ontogenetic stage structure had greater differences among samples within the same fragment than among fragments, i.e., it varied in small spatial scales. The spatial heterogeneity of environmental factors (differences in vegetation cover that varied from open to dense), different reproductive characteristics ( $R$. montana can reproduce asexually, whereas $X$. aromatica reproduces only by seeds) and phenological patterns (annual in $X$. aromatica, and supra-annual in $R$. montana) could be the main variables associated to the ontogenetic structure variation, and they would act differently upon each species in each site. Thus, each site of "cerrado" constitutes a different environment, which can differently influence the success of a species.
\end{abstract}

Key words - “cerrado", ontogenetic stages, Roupala montana, size structure, Xylopia aromatica

RESUMO - (Estrutura populacional de Xylopia aromatica (Lam.) Mart. e de Roupala montana Aubl. em fragmentos de cerrado no Estado de São Paulo). Para entender como a estrutura populacional de espécies diferentes varia entre fragmentos e dentro de um mesmo fragmento, estudamos as distribuições de tamanho e de estádios ontogenéticos de duas espécies comuns de cerrado, em quatro fragmentos de cerrado com diferentes fisionomias e em três locais dentro de um mesmo fragmento com fisionomias semelhantes, no Município de Itirapina ( $22^{\circ} 15^{\prime} \mathrm{S}, 47^{\circ} 48^{\prime} \mathrm{W}$ ), estado de São Paulo, SE Brasil. Medimos a altura e o diâmetro basal do caule (DAS) de todos os indivíduos com DAS $\geq 3 \mathrm{~cm}$ nas seis amostras de 200 parcelas contíguas de 5 x 5 $\mathrm{m}$. Indivíduos com DAS $<3 \mathrm{~cm}$ foram amostrados em sub-parcelas de $1 \mathrm{~m}^{2} \mathrm{em}$ um dos vértices da parcela. Xylopia aromatica apresentou maiores proporções de estádios ontogenéticos iniciais em fisionomias mais abertas, enquanto Roupala montana apresentou maior proporção de estádios iniciais e maior razão jovens:adultos em fisionomias mais densas. Diferenças na estrutura ontogenética foram maiores entre amostras dentro do mesmo fragmento que entre fragmentos diferentes, ou seja, elas variaram em pequenas escalas espaciais. A heterogeneidade espacial das variáveis ambientais (diferenças na cobertura vegetal), as características reprodutivas das espécies ( $R$. montana pode se reproduzir assexuadamente, enquanto $X$. aromatica se reproduz apenas por sementes) e os padrões fenológicos (anual em $X$. aromatica e supra-anual em $R$. montana) parecem ser as principais variáveis associadas à variação das estruturas ontogenéticas e podem atuar de forma diferente nas espécies em cada local. Assim, cada área de cerrado constitui um ambiente diferenciado que pode influenciar o sucesso de uma espécie.

Palavras-chave - cerrado, estádios ontogenéticos, estrutura de tamanho, Roupala montana, Xylopia aromatica

\section{Introdução}

As diferenças de tamanho e de estádios ontogenéticos entre indivíduos de uma população, a forma como os indivíduos estão distribuídos no espaço e as diferenças

\footnotetext{
1. Programa de Pós-Graduação em Biologia Vegetal da Universidade Estadual de Campinas, Instituto de Biologia, Caixa Postal 6109, 13083-970 Campinas, SP, Brasil.

2. Universidade Estadual de Campinas, Instituto de Biologia, Departamento de Botânica, Caixa Postal 6109, 13083-970 Campinas, SP, Brasil.

3. Autor para correspondência: fsantos@unicamp.br
}

genéticas e etárias caracterizam as estruturas populacionais em plantas (Hutchings 1998). Considera-se que as estruturas populacionais sejam influenciadas pela ação de fatores bióticos e/ou abióticos sobre as taxas de crescimento e mortalidade, bem como por eventos passados de recrutamento (Hutchings 1998, Hay et al. 2000). Assim, a caracterização tanto do ambiente quanto da estrutura de populações de plantas é importante para fornecer informações sobre os fatores que influenciam os processos populacionais, a regeneração (Clark 1994) e as respostas de uma população às perturbações em um determinado local (Harper 1977). 
Os fatores que influenciam os processos populacionais variam no espaço em diferentes escalas e, em decorrência, as estruturas das populações podem variar no espaço (Swaine et al. 1987). Sarukhán (1974) e Fowler \& Antonovics (1981) já ressaltavam que a estrutura de populações de plantas pode ser induzida simplesmente pela heterogeneidade espacial existente no ambiente e, assim, pode variar de acordo com a escala de estudo. No entanto, muitos estudos desconsideram a variação espacial nos processos populacionais, chegando a conclusões que não podem ser extrapoladas para escalas diferentes (Moloney 1986).

Alguns autores têm observado que uma mesma espécie pode apresentar diferenças na estrutura populacional entre diferentes sítios e entre locais diferentes dentro de um mesmo sítio (Ramirez \& Arroyo 1990, Milton et al. 1993, Grau 2000). Isso indica que entre fragmentos de mesmo tipo de vegetação podem ser encontradas diferenças na estrutura populacional de uma espécie. Além disso, as características das espécies, principalmente as reprodutivas, têm sido indicadas como influentes na sua estrutura populacional (Oliveira \& Silva 1993, Hoffmann 1999). Hoffmann (1999) observou que espécies com propagação vegetativa apresentavam maior taxa de crescimento populacional e menor taxa de mortalidade do que espécies sem esse tipo de reprodução. Oliveira \& Silva (1993) observaram que espécies que apresentam maior sucesso reprodutivo, em função do seu padrão fenológico, também possuem um aumento no tamanho populacional.

Neste trabalho, avaliamos a estrutura de tamanho e de estádios ontogenéticos de duas espécies perenes, iteropáricas e abundantes em áreas de cerrado do Estado de São Paulo, Roupala montana Aubl. (Proteaceae) e Xylopia aromatica (Lam.) Mart (Annonaceae) em fragmentos de cerrado com diferentes fisionomias e em áreas de cerrado com fisionomias semelhantes dentro de um mesmo fragmento. O objetivo deste trabalho foi entender se e como a estrutura populacional de espécies diferentes varia entre fragmentos com fisionomias diferentes e entre sítios de um mesmo fragmento. Considerando que as duas espécies são intolerantes à sombra (Felfili \& Silva 1992), espera-se que as populações apresentem maior densidade e maior proporção de indivíduos menores e de estádios iniciais em áreas mais abertas. Além disso, considerando que Roupala montana apresenta propagação vegetativa (Hoffmann 1998), ao contrário de Xylopia aromatica (Almeida et al. 1998), espera-se que existam diferenças na estrutura populacional entre as espécies em um mesmo local.

\section{Material e métodos}

Área de estudo - O estudo foi realizado em quatro fragmentos de cerrado no Município de Itirapina, Estado de São Paulo:

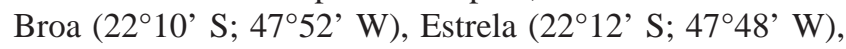

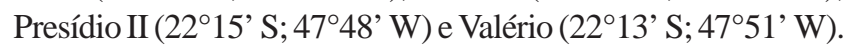
As duas primeiras áreas são particulares, a terceira é uma área de segurança do Presídio João Batista de Arruda Sampaio e a última é administrada pelo Instituto Florestal do Estado de São Paulo, na Estação Experimental de Itirapina (figura 1). O clima da região é do tipo Cwa de Köppen, temperado macrotérmico com inverno seco não rigoroso, com precipitação anual média de $1.425 \mathrm{~mm}$, concentrada no período de outubro a maio, temperatura média de $19,7^{\circ} \mathrm{C}$ e déficit hídrico de 23 mm anuais (Delgado 1994). O solo de cada fragmento é classificado como Neossolo Quartzarênico álico, que é ácido, tem deficiência de nutrientes e teores considerados tóxicos de alumínio trocável (Dutra-Lutgens 2000). Os fragmentos apresentam fisionomias que variam de cerrado stricto sensu ralo (Estrela) até cerrado stricto sensu denso (Valério), com os fragmentos Broa e Presídio II apresentando cobertura arbórea intermediária, segundo o sistema de classificação de Ribeiro \& Walter (1998) (tabela 1).

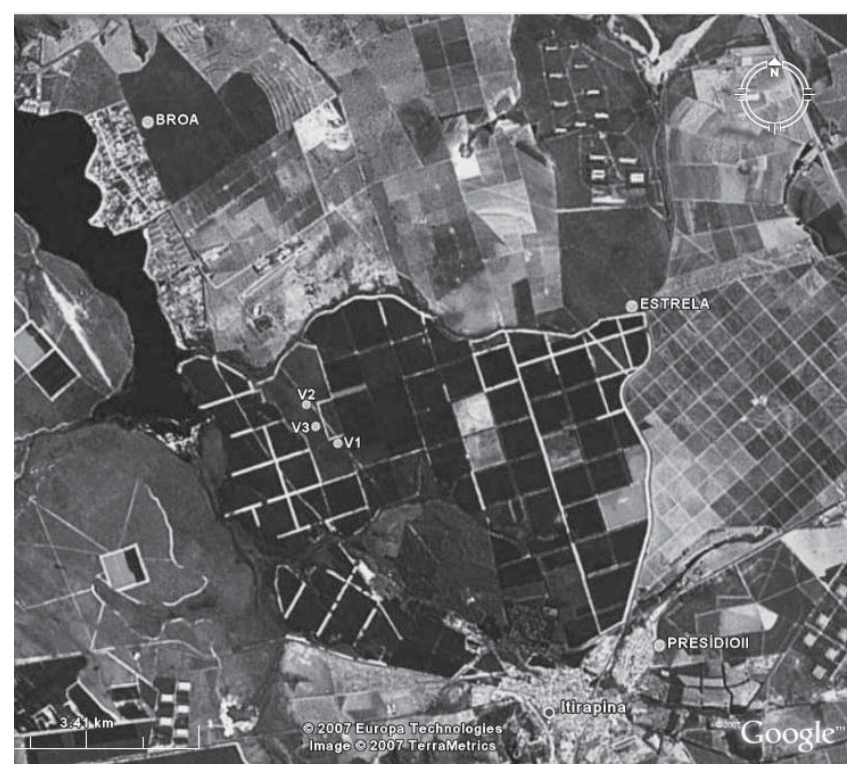

Figura 1. Imagem de satélite da micro-região de Itirapina/SP, com a localização dos fragmentos de cerrado estudados. Broa

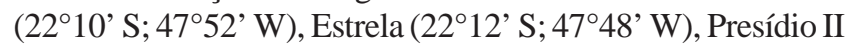
( $\left.22^{\circ} 15^{\prime} \mathrm{S} ; 47^{\circ} 48^{\prime} \mathrm{W}\right)$ e V1 $\left(22^{\circ} 13^{\prime} \mathrm{S}\right.$; $\left.47^{\circ} 51^{\prime} \mathrm{W}\right)$, V2 (22 ${ }^{\circ} 12^{\prime} \mathrm{S}$; $\left.47^{\circ} 51^{\prime} \mathrm{W}\right)$ e V3 $\left(22^{\circ} 12^{\prime} \mathrm{S}\right.$; $\left.47^{\circ} 51^{\prime} \mathrm{W}\right)$ dentro do fragmento Valério (Fonte: Google Earth v.4.2).

Figure 1. Satellite image of the municipality of Itirapina, SE Brazil, with the location of the cerrado fragments studied.

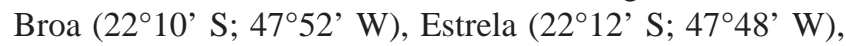
Presídio II $\left(22^{\circ} 15^{\prime} \mathrm{S} ; 47^{\circ} 48^{\prime} \mathrm{W}\right)$ and the areas V1 $\left(22^{\circ} 13^{\prime} \mathrm{S}\right.$;

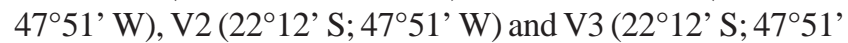
W) within the Valério fragment (Font: Google Earth v.4.2). 
Tabela 1. Fisionomia dos fragmentos estudados no Município de Itirapina, SP.

Table 1. Physiognomy of the cerrado fragments studied in the Municipality of Itirapina, São Paulo State, SE Brazil.

\begin{tabular}{|c|c|}
\hline ÁREA & VEGETAÇÃO \\
\hline $\begin{array}{l}\text { VALÉRIO } \\
(\sim 139,1 \text { ha })\end{array}$ & $\begin{array}{l}\text { Cerrado denso, altura média das árvores de } \\
8 \mathrm{~m} \text {, estrato graminóide ausente e espessa } \\
\text { camada de serapilheira sobre o solo. }\end{array}$ \\
\hline $\begin{array}{l}\text { BROA } \\
(\sim 268,5 \text { ha })\end{array}$ & $\begin{array}{l}\text { Cerrado típico, altura média das árvores de } \\
3 \text { a } 6 \text { m e estrato graminóide presente. }\end{array}$ \\
\hline $\begin{array}{l}\text { PRESÍDIO II } \\
(\sim 12,7 \text { ha })\end{array}$ & $\begin{array}{l}\text { Cerrado típico, altura média das árvores de } \\
3 \text { a } 6 \text { m e estrato graminóide presente. }\end{array}$ \\
\hline $\begin{array}{l}\text { ESTRELA } \\
(\sim 212,1 \text { ha })\end{array}$ & $\begin{array}{l}\text { Cerrado ralo, altura média das árvores de } 2 \\
\text { a } 4 \mathrm{~m} \text {, estrato graminóide presente. }\end{array}$ \\
\hline
\end{tabular}

As espécies - Xylopia aromatica é árvore típica do cerrado stricto sensu, podendo ocorrer no cerradão. A espécie é pioneira, geralmente ocorrendo em áreas perturbadas, como beira de estradas ou clareiras, sendo abundante em cerrados com alta luminosidade (Almeida et al. 1998). Apresenta alta capacidade de rebrota após a eliminação da parte aérea por fogo ou por geada (Silberbauer-Gottsberger et al. 1977). Estudos fenológicos realizados em Itirapina mostraram período de floração de novembro a abril e de frutificação de dezembro a maio (Almeida et al. 1998), mas foram observadas floração e frutificação dessa espécie em todos os meses do ano na área estudada (Miranda-Melo, observação pessoal).

Roupala montana pode ocorrer em quase todas as fisionomias de cerrado, desde o campo limpo até o cerradão, ocorrendo principalmente em cerrado stricto sensu típico, cerrado stricto sensu ralo e campo limpo. Essa espécie reproduz-se vegetativamente por produção de raízes gemíferas (Hoffmann 1998), mas também apresenta a capacidade de rebrotar, principalmente quando danificada pelo fogo (Hoffmann \& Solbrig 2003). Apresenta padrões supra-anuais de floração e/ou frutificação (Borges 2000).

Procedimento - Em cada fragmento foi instalada uma grade de 50 x 100 m, subdividida em 200 parcelas contíguas de 5 x 5 $\mathrm{m}$, totalizando 0,5 ha. No fragmento do Valério, foram instaladas grades em três sítios, denominados V1, V2 e V3, separadas por uma distância de pelo menos $500 \mathrm{~m}$. Em cada parcela foram marcados todos os indivíduos de Xylopia aromatica e Roupala montana com diâmetro do caule à altura do solo $(\mathrm{DAS}) \geq 3 \mathrm{~cm}$. Em uma parcela de $1 \mathrm{~m}^{2}$, sorteada dentre os vértices de cada parcela de $25 \mathrm{~m}^{2}$, foram marcados os indivíduos com DAS $<3 \mathrm{~cm}$. A altura e o DAS de todos os indivíduos marcados foram medidos entre julho e setembro de 2002
Os indivíduos foram classificados em seis estádios ontogenéticos (Miranda-Melo, dados não publicados), identificados como plântula (PL, com uma ou duas folhas cotiledonares), jovem 1 (J1, sem cotilédones e sem ramificações), jovem 2 (J2, com ramificações), imaturo ( $\mathrm{Im}$, com ramificações e reiterações), adulto vegetativo (AV, com diâmetro e altura iguais ou maiores que os do menor indivíduo reprodutivo, mas que não se reproduziu no período de coleta de dados) e adulto reprodutivo (AR, com flor, fruto ou cicatriz dessas estruturas).

Os fragmentos, de acordo com a sua classificação fisionômica, apresentam diferentes aberturas de dossel, com o Estrela sendo considerado o fragmento mais aberto, o Broa e o Presídio II considerados como possuindo aberturas de dossel intermediárias e o Valério considerado como o mais fechado. Entretanto, diferentes localidades dentro de um mesmo fragmento podem apresentar diferenças de abertura do dossel. Para verificar esse aspecto, foram obtidas fotografias hemisféricas no Valério, no centro de cada parcela de $25 \mathrm{~m}^{2}$ em V1, V2 e V3, com uma câmera digital Nikon Coolpix 950 e lente olho-de-peixe autofocus Nikon $8 \mathrm{~mm}$ com ângulo de $180^{\circ}$ colocada a $1,30 \mathrm{~m}$ acima do solo.

Análise de dados - A estrutura ontogenética foi analisada por meio da distribuição de freqüência dos indivíduos nos diferentes estádios ontogenéticos. As distribuições de estádios ontogenéticos foram comparadas entre os fragmentos e entre as espécies através do teste de Kolmogorov-Smirnov (Zar 1999), com correções de Bonferroni para testes seqüenciais (Rice 1989). As estruturas de tamanho (diâmetro e altura) foram analisada através do coeficiente de Gini (Weiner \& Solbrig 1984). Foi feito um teste de bootstrap com 1.000 repetições para verificar diferenças dos coeficientes entre fragmentos e espécies. As análises foram feitas utilizando-se o programa WINGINI versão 1.0 (Santos 1996).

As porcentagens de abertura do dossel foram obtidas com o programa Gap Light Analyzer versão 2.0 (Frazer et al. 1999). Posteriormente, esses dados foram transformados em arco-seno da raiz quadrada da porcentagem, para comparar a abertura do dossel entre localidades no fragmento Valério através do teste de Kruskal-Wallis (Zar 1999).

\section{Resultados}

As áreas do Valério diferiram quanto à abertura do dossel. A porcentagem de abertura do dossel no Valério 1 foi a menor em relação às demais áreas analisadas $(H=364,751 ; g l=2 ; P<0,001)$ com valor médio de $19,6 \%$. Valério 2 e 3 apresentaram aberturas semelhantes do dossel $(P=0,187)$, sendo os valores médios de abertura $29,4 \%$ e $28,6 \%$, respectivamente.

As distribuições de estádios ontogenéticos de Xylopia aromatica diferiram entre os fragmentos $\left(\mathrm{D}_{\text {máx }}\right.$ $=0,06$ a 0,$53 ; P<0,01)$, exceto entre o Estrela e o Presídio II $\left(\mathrm{D}_{\text {máx }}=0,15 ; P>0,05\right)$. Nas três áreas do 
Valério, $X$. aromatica apresentou maior proporção de $\mathrm{J} 2$, embora com diferenças entre as áreas. No Broa, $X$. aromatica apresentou predomínio de $\mathrm{J} 1$, com cerca de $60 \%$ dos indivíduos nesse estádio. No Estrela e no Presídio II as proporções de J1 e J2 foram similares (cerca de $43 \%$ de $\mathrm{J} 1$ e $36 \%$ de $\mathrm{J} 2$ no Estrela e $48 \%$ para ambos no Presídio II), mas nenhum AV e poucos Im foram amostrados nesses fragmentos (figura 2).

Roupala montana ocorreu somente em dois fragmentos e em V1 apresentou 6,4 vezes mais indivíduos que no Broa (figura 2). A distribuição de estádios ontogenéticos diferiu entre os fragmentos $\left(D_{\text {máx }}=0,32\right.$; $P<0,01)$, apresentando maior freqüência de $\mathrm{J} 1 \mathrm{em} \mathrm{V} 1$ (80\%) que no Broa (47\%).

A distribuição dos estádios ontogenéticos diferiu entre as espécies em V1 $\left(\mathrm{D}_{\text {máx }}=0,73, P<0,01\right)$ e no
Broa $\left(\mathrm{D}_{\text {máx }}=0,12, P<0,01\right)$. Em V1 (figura 2), R. montana apresentou aproximadamente quatro vezes mais indivíduos e maior proporção de $\mathrm{J} 1(80 \%)$ do que $X$. aromatica $(7,5 \%)$. No Broa, apesar de ambas as espécies apresentarem maior proporção de $\mathrm{J} 1$ do que os demais estádios, $X$. aromatica apresentou 1,3 vezes mais $\mathrm{J} 1$ do que $R$. montana.

A distribuição de diâmetro de $X$. aromatica foi semelhante no V1 (Gini = 0,621), no V2 (Gini =0,632), no V3 (Gini $=0,619)$, no Estrela $($ Gini $=0,638)$ e no Presídio II (Gini $=0,669)$, todos $\operatorname{com} P>0,05$. A distribuição de altura de $R$. montana foi semelhante no V1 (Gini $=0,542)$ e no Broa (Gini $=0,551)$, $\operatorname{com} P=$ 0,48 . No V1, as distribuições de tamanho entre as espécies foram semelhantes considerando tanto o diâmetro (Gini $=0,621$ em $R$. montana e em $X$.

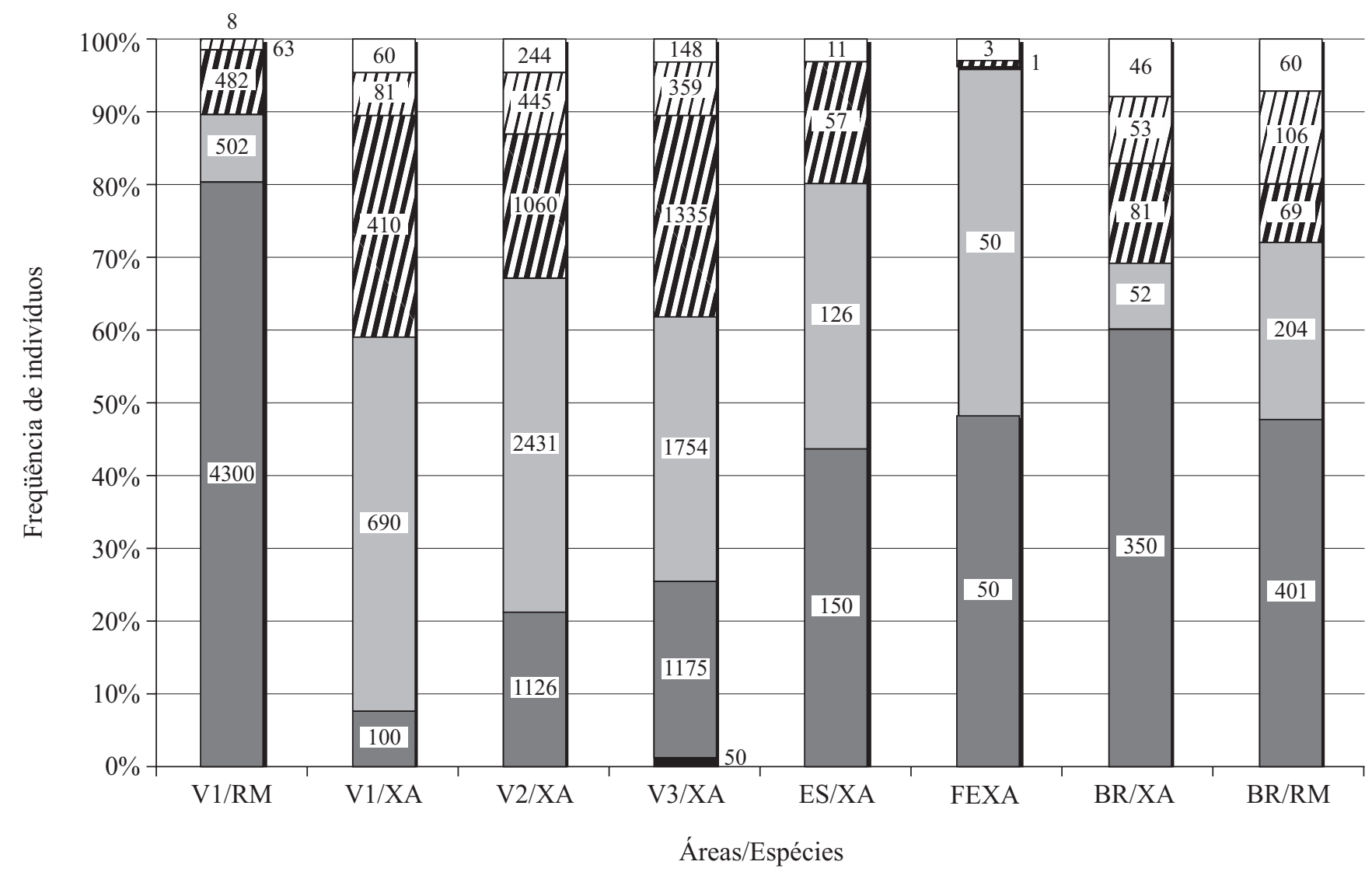

Figura 2. Distribuição (porcentagem e número) de indivíduos amostrados de cada estádio ontogenético de Xylopia aromatica (XA) e Roupala montana (RM) nos sítios de cerrado estudados (V1 = Valério 1, V2 = Valério 2, V3 = Valério 3, BR = Broa, $\mathrm{ES}=$ Estrela, $\mathrm{FE}=$ Presídio II, $\square \mathrm{AR}=$ Adulto reprodutivo, $\mathrm{mWI} \mathrm{AV}=$ Adulto vegetativo, $\mathrm{IIII} \mathrm{IM}=\mathrm{Imaturo}, \square \mathrm{J} 2=\mathrm{Jovem} 2$, $\square \mathrm{J} 1$ = Jovem 1, $\square$ PL = Plântula).

Figure 2. Distribution (percentage and number) of sampled individuals of each ontogenetic stages of Xylopia aromatica (XA) and Roupala montana $(\mathrm{RM})$ in the sites studied $(\mathrm{V} 1=$ Valério $1, \mathrm{~V} 2=$ Valério 2, V3 = Valério 3, BR = Broa, ES = Estrela,

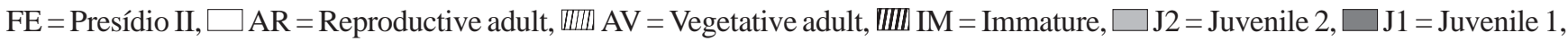
PL $=$ Seedling). 
aromatica, $P=0,90)$ quanto a altura (Gini $=0,546$ para $R$. montana e 0,526 para $X$. aromatica, $P=0,07)$. No Broa, as distribuições de tamanho diferiram entre as espécies $(P<0,001)$, com $X$. aromatica apresentando maior desigualdade de tamanhos (Gini $=0,733$ para diâmetro e 0,647 para altura) do que $R$. montana (Gini $=0,571$ para diâmetro e 0,551 para altura).

\section{Discussão}

A estrutura ontogenética diferiu entre as espécies, bem como entre fragmentos e entre locais dentro de um mesmo fragmento. Diferenças na estrutura ontogenética foram maiores dentro de um mesmo fragmento que entre fragmentos diferentes. No entanto, as estruturas de tamanho, na sua maioria, não diferiram significativamente.

Outros autores também têm verificado variação na estrutura populacional entre áreas e associaram essa variação a picos no recrutamento. Esses autores argumentaram que variações temporais no recrutamento entre áreas podem ocorrer devido a condições climáticas, formação de clareiras, flutuação no período de produção de sementes, predação de sementes ou herbivoria, podendo explicar diferenças no estabelecimento de uma espécie entre áreas (Ramirez \& Arroyo 1990, Grau 2000) e gerar variações espaciais nas estruturas populacionais. No caso de Roupala montana e Xylopia aromatica, é possível que as diferentes proporções de J1 e J2 encontradas entre as áreas não sejam decorrentes de diferenças climáticas, uma vez que os fragmentos são relativamente próximos e estão sujeitos ao mesmo clima regional. Como esperado, X. aromatica apresentou maior proporção de estádios ontogenéticos nos sítios de fisionomia mais aberta. Porém, R. montana apresentou maior proporção de estádios iniciais no sítio de fisionomia mais densa, ao contrário do esperado. Assim, diferentes variáveis, além de variações estocásticas, poderiam influenciar na estrutura populacional e provocar sua variação no espaço.

Diferenças na fisionomia da vegetação e variáveis a ela associadas podem influir na estrutura populacional. É sabido que a heterogeneidade do habitat pode alterar as chances de crescimento e de sobrevivência de uma planta, dependendo de sua posição no espaço (Hutchings 1998). A luz é reconhecidamente muito variável em intensidade e qualidade no espaço e no tempo (Hutchings 1998), podendo ser influenciada pela cobertura vegetal. Além disso, a luz é essencial na fase inicial de crescimento, principalmente para espécies intolerantes à sombra, como X. aromatica e R. montana (Felfili \& Silva 1992).
A estrutura ontogenética das populações que estudamos pode ser influenciada por variações na intensidade e na qualidade de variáveis ambientais no espaço, resultando em diferenças nas proporções de juvenis, ocasionando ora maior proporção de $\mathrm{J} 1$ ora de $\mathrm{J} 2$. No entanto, as diferenças de estrutura ontogenética não produziram grandes diferenças na estrutura de tamanho entre as populações.

As diferenças de estrutura ontogenética entre $R$. montana e $X$. aromatica decorreram principalmente de variações na proporção de juvenis. Segundo Cook (1983), a alta proporção de juvenis pode ser um indicativo de predominância de propagação vegetativa numa população. Estudos têm destacado que a propagação vegetativa pode levar a espécie a garantir a sua permanência no ambiente, afetando a dinâmica de regeneração da vegetação (Hoffmann 2002). Além disso, Hoffmann (1998, 1999), em trabalhos com $R$. montana, destacou que indivíduos produzidos por propagação vegetativa geralmente possuem menor taxa de mortalidade do que plântulas de idade similar e tendem a alcançar a maturidade sexual mais cedo, podendo contribuir para o aumento da taxa de crescimento populacional, mesmo quando a reprodução sexual tem sua importância diminuída.

A limitação da reprodução sexuada em plantas lenhosas tem como conseqüência o aumento da importância relativa da propagação vegetativa na manutenção das populações (Hoffmann 1998, 1999) e tem sido atribuída a distúrbios, principalmente a queimadas freqüentes. Não temos o histórico das queimadas nos sítios estudados em Itirapina e não podemos concluir sobre sua influência na variação da estrutura das populações. No entanto, outros pesquisadores consideraram fatores bióticos como os principais fatores limitantes ao tamanho das populações. Oliveira \& Silva (1993) indicaram que os processos fisiológicos e a susceptibilidade à predação de frutos de duas espécies de cerrado seriam os principais fatores limitantes de sua reprodução e sugeriram que as interações biológicas teriam papel mais importantes na dinâmica populacional do que as restrições ambientais.

Diferenças no padrão fenológico entre espécies também têm sido ressaltadas como geradoras de diferentes estruturas populacionais. Roupala montana apresenta intervalos supra-anuais de floração e episódios de frutificação massiva (Borges 2000), enquanto Xylopia aromatica floresce e frutifica anualmente (Almeida et al. 1998). A ocorrência desses eventos num passado recente pode também ter contribuído para a geração das diferenças observadas na estrutura populacional das duas espécies. 
As diferenças na estrutura populacional de $X$. aromatica e $R$. montana foram maiores dentro de um mesmo fragmento do que entre fragmentos, ou seja, variaram mais em pequenas escalas espaciais. Esse fato pode indicar que o tipo de reprodução, o padrão fenológico e a heterogeneidade espacial de variáveis ambientais podem atuar como fatores associados às variações das populações e que sua influência relativa varia no espaço.

Agradecimentos - À FAPESP, pela bolsa de Mestrado para A.A. Miranda-Melo (Proc. no. 01/13046-1). Ao Conselho Nacional de Desenvolvimento Científico e Tecnológico (CNPq), pela bolsa de produtividade em pesquisa (307132/ 2004-8) para F.A.M. Santos. Ao Instituto Florestal, pelo apoio logístico ao desenvolvimento do estudo. Ao Instituto Arruda Botelho, pela autorização deste estudo nos sítios do Broa e do Estrela.

\section{Referências bibliográficas}

ALMEIDA, S.P., PROENÇA, C.E., SANO, S.M. \& RIBEIRO, J.F. 1998. Cerrado: espécies vegetais úteis. Embrapa, Planaltina.

BORGES, H.B.N. 2000. Biologia reprodutiva e conservação do estrato lenhoso numa comunidade do cerrado. Tese de doutorado, Universidade Estadual de Campinas, Campinas.

CLARK, D.A. 1994. Plant demography. In La Selva - ecology and natural history of a neotropical rain forest (L.A. Mc Dade, K.S. Bawa, H.A. Hespenheide \& G.S. Hartshorn, eds.). The University of Chicago Press, Chicago, p.90-105.

COOK, R.E. 1983. Clonal plant populations. American Scientist 71:244-253.

DELGADO, J.M. 1994. Plano de manejo integrado das unidades de Itirapina-SP. Instituto Florestal de São Paulo, São Paulo.

DUTRA-LUTGENS, H. 2000. Caracterização ambiental e subsídios para o manejo da zona de amortecimento da Estação Experimental e Ecológica de Itirapina - SP. Dissertação de mestrado, Universidade Estadual Paulista Júlio de Mesquita Filho, Rio Claro.

FELFILI, J.M. \& SILVA JÚNIOR, M.C. 1992. Floristic composition, phytosociology and comparison of cerrado and gallery forests at Fazenda Agua Limpa, Federal District, Brazil. In Nature and dynamics of the forestsavanna boundaries (P.A. Furley, J. Procter \& J.A. Ratter, eds.). Chapman \& Hall, London, p.393-429.

FOWLER, N. \& ANTONOVICS, J. 1981. Small-scale variability in the demography of transplants of two herbaceous species. Ecology 62:1450-1457.

FRAZER, G.W., CANHAM, C.D. \& LERTZMAN, K.P. 1999. Gap Light Analyzer (GLA), Version 2.0. Imaging software extract canopy structure and gap light transmission indices from true-colour fisheye photographs, users manual and program documentation. Simon Fraser University, Burnaby, British Columbia, and The Institute of Ecosystem Studies, Millbrook, New York.
GRAU, H.R. 2000. Regeneration patterns of Cedrella lilloi (Meliaceae) in northwestern Argentina subtropical montane forests. Journal Tropical Ecology 16:227242.

HARPER, J.L. 1977. Population biology of plants. Academic Press, London.

HAY, J.D., BIZERRIL, M.X., CALOURO, A.M., COSTA, E.M.N., FERREIRA, A.A., GASTAL, M.L.A., GOES JÚNIOR, C.D., MANZAN, D.J., MARTINS, C.R., MONTEIRO, J.M.G., OLIVEIRA, S.A., RODRIGUES, M.C.M., SEYFARTH, J.A.S. \& WALTER, B.M.T. 2000. Comparação do padrão da distribuição espacial em escalas diferentes de espécies ativas do cerrado, em Brasília, DF. Revista Brasileira de Botânica 23:341347.

HOFFMANN, W.A. 1998. Post-burn reproduction of woody plants in a neotropical savanna: the relative importance of sexual and vegetative reproduction. Journal of Applied Ecology 35:422-433.

HOFFMANN, W.A. 1999. Fire and population dynamics of woody plants in a neotropical savanna: matrix model. Ecology 80:1354-1369.

HOFFMANN, W.A. 2002. Direct and indirect effects of fire on radial growth of cerrado savanna trees. Journal of Tropical Ecology 18:137-142.

HOFFMANN, W.A. \& SOLBRIG, O.T. 2003. The role of topkill in the differential response of savanna woody species to fire. Forest Ecology and Management 180:273-286.

HUTCHINGS, M.J. 1998. Structure of plant population. In Plant Ecology (M.J. Crawley, ed.). Blackwell Scientific Publications, Oxford, p.325-358.

MILTON, S.J., RYAN, P.G., MOLONEY, C.L., COOPER, J. \& DEAN, W.R.J. 1993. Disturbance and demography of Phylica arbores (Rhamnaceae) on the Tristan-Gough group of islands. Botanical Journal of Linnean Society 111:55-70.

MOLONEY, K.A. 1986. Wave and non-wave regeneration processes in a subalpine Abies balsamea forest. Canadian Journal of Botany 61:3337-3342.

OLIVEIRA, P.E. \& SILVA, J.C.S. 1993. Reproductive biology of two species of Kielmeyera (Guttiferae) in the cerrados of central Brazil. Journal of Tropical Ecology 9:67-79.

RAMIREZ, N. \& ARROYO, M.K. 1990. Estrutura populacional de Copaifera pubiflora Benth. (Leguninosae, Caesalpinoideae) em los lhanos centrales de Venezuela. Biotropica 22:124-132.

RIBEIRO, J.F. \& WALTER, B.M.T. 1998. Fitofisionomias do bioma Cerrado. In Cerrado: ambiente e flora (S.M. Sano \& S.P. Almeida, eds.). Embrapa, Planaltina, p.89-166.

RICE, W.R. 1989. Analyzing tables of statistical tests. Evolution 43:223-225.

SANTOS, F.A.M. 1996. Wingini: Programa para cálculo do coeficiente de Gini. Versão 1.0. Departamento de Botânica. Universidade Estadual de Campinas, Campinas. 
SARUKHÁN, J. 1974. Studies on plant demography: Ranunculus repens L., R. bulbosus L., and $R$. acris L. II. Reproductive strategies and seed population dynamics. Journal of Ecology 62:151-177.

SILBERBAUER-GOTTSBERGER, I.; MORAWETZ, W. \& GOTTSBERGER, G. 1977. Frost damage of cerrado plants in Botucatu, Brazil, as related to the geographical distribution of the species. Biotropica 9:253-261.
SWAINE, M.D., LIEBERMAN, D. \& PUTZ, F.E. 1987. The dynamics of tree populations in tropical forest: a review. Journal of Tropical Ecology 3:359-366.

WEINER, J. \& SOLBRIG, O.T. 1984. The meaning and measurement of size hierarchies in plant populations. Oecologia, 61:334-336.

ZAR, J.H. 1999. Biostatistical analysis. Prentice-Hall, New Jersey. 